\title{
GENERACIÓN DE BIOSEÑALES SINTÉTICAS MEDIANTE SERIES DE FOURIER VARIANTES EN EL TIEMPO
}

\author{
J. Escrivá Muñoz ${ }^{*}{ }^{1,2}$, A. Galea Cazorla ${ }^{2}$, C. González Pijuán ${ }^{1,2}$, M. Vallverdú ${ }^{1}$, P. Caminal ${ }^{1}$, E. W. Jensen ${ }^{2}$ \\ ${ }^{1}$ Dep. ESAII, Centre de Recerca en Enginyeria Biomèdica (CREB), Universitat Politècnica de Catalunya, \\ CIBER de Nanomedicina, Biomateriales y Bioingeniería (CIBER-BBN) \\ ${ }^{2}$ Research and Development Department, Quantium Medical SL, Mataró 08302
}

*jem@quantiummedical.com

\begin{abstract}
Resumen
La selección de la técnica más adecuada para el análisis de una señal tiempo-frecuencia depende en gran medida de la propia naturaleza de la señal objeto de análisis. Para ello, resulta adecuado utilizar señales sintéticas con un contenido tiempo-frecuencia conocido. En este trabajo se ha propuesto la construcción de una base de datos de señales biomédicas sintéticas a partir de la clasificación en patrones de señales reales. El objetivo de esta base de datos ha sido disponer de señales sintéticas con características tiempo-frecuencia predeterminadas y modificables con un comportamiento lo más realista posible.
\end{abstract}

Palabras Clave: modelado de señales, análisis tiempo-frecuencia, series de Fourier, impedancia cardiográfica.

\section{INTRODUCCIÓN}

Las técnicas avanzadas de análisis de señales biomédicas y su aplicación automática al diagnóstico son cada vez más utilizadas en los equipos clínicos. En este campo, la inspección visual de las señales biomédicas puede ser un proceso tedioso, especialmente en los registros de larga duración.

Las señales biológicas pueden presentar un comportamiento periódico o cuasi periódico bajo ciertas circunstancias, por lo que la representación de la periodicidad es importante en el procesado de señales en las aplicaciones biomédicas. Los métodos frecuenciales ofrecen una representación compacta de estas señales en el dominio de la frecuencia, siendo un método simple pero ampliamente aceptado. Sin embargo, muchas señales biológicas tienen un comportamiento variable en el dominio de la frecuencia dependiendo de cada instante temporal. En estos casos, es interesante analizar cómo la distribución frecuencial cambia con el tiempo.
El análisis mediante distribuciones tiempo-frecuencia (DTF) se aplica frecuentemente al estudio de electroencefalogramas (EEG) [6], variabilidad del ritmo cardiaco (VRC) [7], señales de voz patológicas, etc. [2]. Son varios los núcleos (del inglés, kernel) de DTF válidos para el análisis de las bioseñales, pero su aplicación requiere alta claridad, la ausencia de términos cruzados interferentes, el cumplimiento de ciertas propiedades matemáticas y baja complejidad computacional [3]. No obstante, la elección de un núcleo apropiado depende de la propia naturaleza de la señal a analizar. Debido a la complejidad de estas señales, es frecuente utilizar señales sintéticas para evaluar la bondad de las DTF. En este sentido, el presente trabajo propone una metodología para diseñar señales sintéticas con los siguientes requisitos: a) las señales sintéticas deben ser muy similares a las señales originales tanto en su contenido temporal como frecuencial; b) sus parámetros tiempofrecuencia deben ser fácilmente modificables.

Con el fin de lograr estos requisitos, se ha propuesto analizar el latido-patrón más frecuente en señales reales de impedancia cardiográfica para generar un modelo con series de Fourier. Este modelo paramétrico basado en una serie de Fourier será utilizado para crear señales con variaciones conocidas en el dominio tiempo-frecuencia.

\section{METODOLOGÍA}

\subsection{SEÑALES DE IMPEDANCIA CARDIOGRÁFICA}

Las señales reales de impedancia cardiográfica $(Z \mathrm{O}$ ICG) se obtuvieron de una muestra de datos proporcionada por Quantium Medical, S.L. (Barcelona). Estas señales fueron registradas utilizando el monitor qCO, el cual utiliza la información de la curva de impedancia para el cálculo de diferentes variables hemodinámicas. Este monitor registra la señal ICG mediante 4 electrodos, con un par de ellos inyectando una corriente constante $(400 \mu \mathrm{A}$ RMS a $50 \mathrm{kHz}$ ), y un segundo par de electrodos 
midiendo la tensión resultante. La tensión es amplificada y digitalizada con una frecuencia de muestreo de $250 \mathrm{~Hz}$.

Los cambios en la señal de impedancia torácica representan variaciones en el flujo de sangre. La señal $\mathrm{Z}$ original se deriva en $-\mathrm{dZ} / \mathrm{dt}$ con el fin de enfatizar los puntos de inflexión de la señal Z .

Para este estudio, esta señal es post-procesada aplicando un filtro paso bajo de $30 \mathrm{~Hz}$ con el fin de reducir ruido, tanto en las direcciones de avance como de retroceso para evitar la distorsión de fase cero.

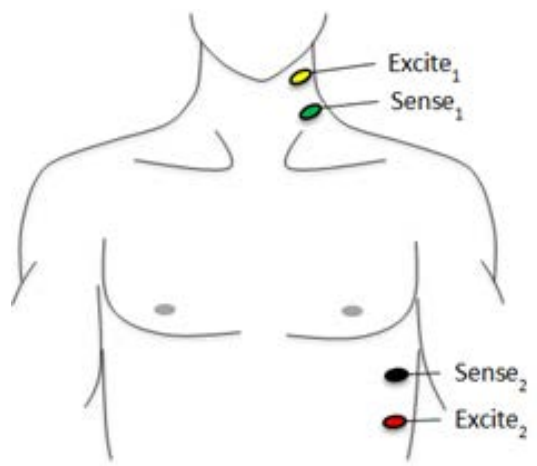

Figura 1: Ubicación de los electrodos qCO [9]

\subsection{RECONOCIMIENTO DE PATRONES}

Se propone una metodología para el reconocimiento de patrones que permita detectar las formas de onda más típicas contenidas en las señales ICG. Se aísla cada latido del ICG y se normaliza para obtener media cero y desviación estándar unitaria, siendo la longitud de cada ventana de 200 muestras. Los puntos de inicio $\mathrm{y}$ fin se definen como los puntos de mínima amplitud entre dos ICG máximos. El valor máximo de ICG es localizado utilizando los picos QRS del electrocardiograma (ECG), mediante un detector de Pan-Tompkins [10].

Seguidamente, se determina el primer patrón de la base de datos como el primer latido disponible del registro. El siguiente latido se compara con el primer patrón y se calcula su correlación. Si su correlación es mayor de 0.90 , el patrón se actualiza con la media entre el patrón original y el nuevo latido. De lo contrario, se crea un nuevo patrón.

Este método asigna un patrón a cada latido del ICG. Cada latido ICG se promedia con el patrón de la base de datos de patrones con el que muestre mayor correlación (por encima del umbral $t h_{\text {corr }}$ ), teniendo en cuenta los pesos relativos de los latidos y el patrón en el promediado. Si no se alcanza el umbral de correlación, se crea un nuevo patrón. La metodología seguida para el reconocimiento de patrones se halla resumida en la Figura 2 (en la cual el umbral de correlación $t h_{\text {corr }}$ queda abreviado como $t h$ ).

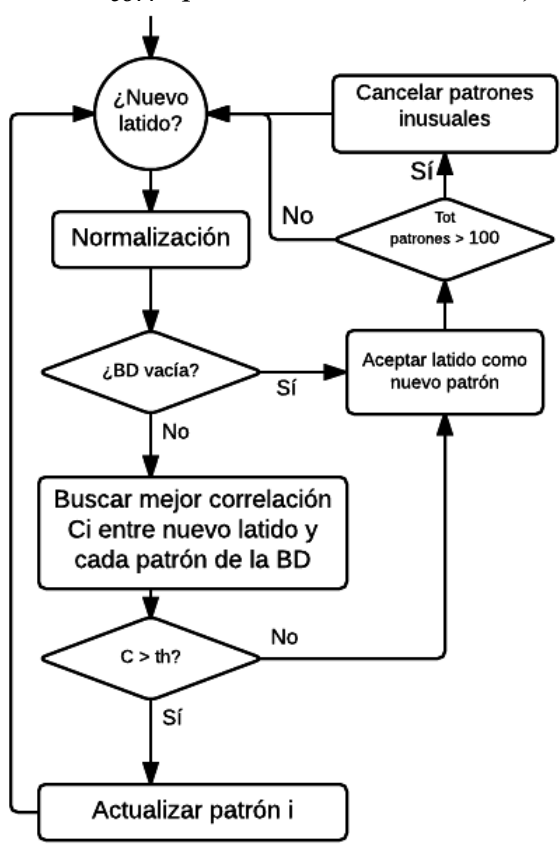

Figura 2: Metodología del reconocimiento de patrones

\subsection{MODELADO DE FOURIER}

Supóngase que el patrón más frecuente $\tilde{x}(t)$ es un periodo de duración T de una señal $x(t)$ de frecuencia $\Omega_{0}=2 \pi / T$, que existe para todo $t$ y además satisface las condiciones de Dirichlet [4]. Entonces, podemos representar dicha $x(t)$ con una serie de Fourier (1):

$$
\begin{gathered}
x(t)=\sum_{n=0}^{\infty}\left[a_{n} \cos \left(n \Omega_{0} t\right)+b_{n} \sin \left(n \Omega_{0} t\right)\right] \\
=\sum_{n=-\infty}^{\infty} c_{n} e^{j n \Omega_{0} t}
\end{gathered}
$$

donde $a_{n}$ y $b_{n}$ son constantes reales y $c_{n}$ son constantes complejas.

La ecuación (1) indica que la serie de Fourier requiere un número infinito de términos para reproducir con precisión la señal $x(t)$. Si se trunca la serie, se reduce la exactitud de la representación y los mayores errores se producen en la proximidad de los puntos de discontinuidad (fenómeno de Gibbs).

En el dominio discreto, la señal $x[n]$ tendrá una estructura de serie de Fourier de frecuencia $\omega_{n}=$ $2 \pi / N$, donde $N$ será la longitud de un patrón.

\subsection{MODELADO CON VARIACIONES TIEMPO-FRECUENCIA LINEALES Y CUADRÁTICAS}


El modelado de Fourier aplicado al patrón propuesto $\tilde{x}(t)$ permite modificar de forma controlada las características tiempo-frecuencia de la señal temporal completa $x(t)$ de manera similar a cómo varían las características tiempo-frecuencia de señales chirp. Una señal chirp es aquella cuya frecuencia varía de forma conocida. Esta variación frecuencial puede ser:

- Lineal, con una frecuencia de barrido instantánea dada por:

$$
\begin{gathered}
f_{i}(t)=f_{0}+\beta t \\
\beta=\left(f_{1}-f_{0}\right) / 2 t_{1}
\end{gathered}
$$

- Cuadrática, con una frecuencia de barrido instantánea dada por:

$$
\begin{aligned}
& f_{i}(t)=f_{0}+\beta t^{2} \\
& \beta=\left(f_{1}-f_{0}\right) / 3 t_{1}^{2}
\end{aligned}
$$

En ambas, $\beta$ garantiza que se mantiene la frecuencia de corte deseada $f_{1}$ en el tiempo $t_{1}$.

\subsection{MODELADO CON VARIACIONES TIEMPO-FRECUENCIA ALEATORIAS Y CAÓTICAS}

Se consideran también variaciones aleatorias o caóticas en el diseño de $x(n)$. Estas características pueden implementarse latido a latido cambiando la longitud del patrón a través de un proceso de interpolación o de diezmado según una serie predefinida de longitudes RR (en referencia a la distancia entre picos QRS consecutivos en el ECG).

En este trabajo, se diseñan series RR que exhiben comportamientos aleatorios y caóticos. Así, se ha utilizado como serie RR la serie $H_{x}$ obtenida a partir del mapa de Hénon [8]:

$$
\left\{\begin{array}{l}
H_{x}(n+1)=1-a H_{x}^{2}(n)+H_{y}(n) \\
H_{y}(n+1)=b H_{x}(n)
\end{array}\right.
$$

donde a y b son dos constantes reales. Esta última aproximación permite crear una señal de ICG cuya periodicidad cambia de forma caótica.

\subsection{MODELADO CON VARIACIONES RESPIRATORIAS}

Las señales de ICG generalmente presentan fluctuaciones en función de la frecuencia respiratoria. Para una frecuencia cardíaca de 60 latidos por minuto, deben esperarse unas 12 respiraciones por minuto. Por tanto, se genera una nueva señal que tiene en cuenta la fluctuación de la respiración. Esta fluctuación se ha modelado tal como se indica a continuación:

$$
r(t)=-\left|\cos \left(2 \pi f \frac{r}{2} t\right)\right|
$$

donde $f_{r / 2}$ es la mitad de la frecuencia respiratoria, y puede expresarse en relación de la longitud RR tal como:

$$
f_{r / 2}=\frac{1}{2} \cdot \frac{1}{N_{r}}=\frac{1}{2} \cdot \frac{1}{12 N}
$$

donde $N_{r}$ es la duración del ciclo respiratorio y $N$ es la duración de cada latido del ICG.

Esta influencia respiratoria se ha añadido al modelo introduciendo variaciones en los valores de la serie RR. Además, se ha añadido ruido blanco para estudiar diferentes ratios señal-ruido (SNR, del inglés signalto-noise ratio).

\section{RESULTADOS}

\subsection{ANÁLISIS DEL RECONOCIMIENTO DE PATRONES}

Los registros ICG se incluyeron en el estudio con el fin de definir los patrones. Las señales ICG se procesaron con un filtro paso bajo de frecuencia de corte de $30 \mathrm{~Hz}$ y se utilizaron dos umbrales de correlación de 0,85 y 0,90 en dos análisis distintos. En total se analizaron 13.618 latidos de impedancia, determinándose un total de 80 patrones diferentes con ambos umbrales. La Figura 3 muestra los patrones más típicos y su localización a lo largo de los registros para el caso de $\mathrm{Th}_{\text {corr }}=0,90$.

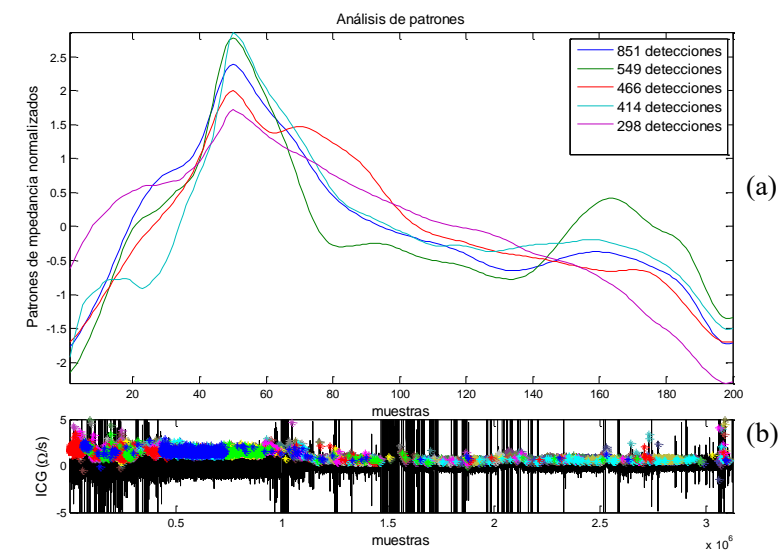

Figura 3: Morfología de los cinco patrones más frecuentes (a) del registro íntegro de ICG donde se indican la aparición de los patrones (b)

La influencia del umbral de correlación se muestra en la Figura 4. El número de patrones con al menos 100 repeticiones es mayor con un umbral $\mathrm{Th}_{\text {corr }}=0,90$ (50 patrones) que con $\mathrm{Th}_{\text {corr }}=0,85$ (37 patrones). Además, en el caso del umbral $\mathrm{Th}_{\text {corr }}=0,90$, el patrón principal 
apareció 851 veces, mientras que en el caso del umbral $\mathrm{Th}_{\mathrm{corr}}=0,85$, el mismo patrón se repitió 1.320 veces.

Para el modelado de Fourier se seleccionó el patrón más frecuente obtenido con un umbral $\mathrm{Th}_{\text {corr }}=0,85$, dado que no se apreciaban diferencias importantes con umbrales mayores. Dicho patrón fue enventanado para suavizar las transiciones entre patrones consecutivos de una serie temporal.

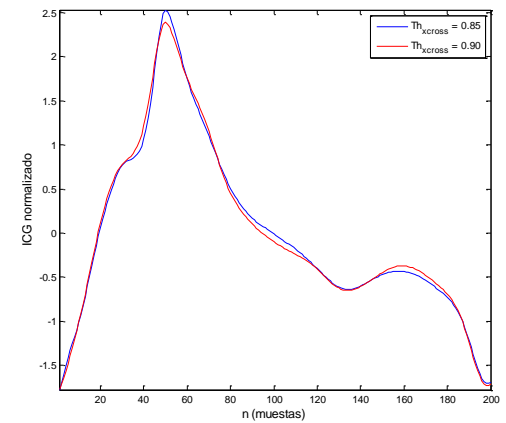

(a)

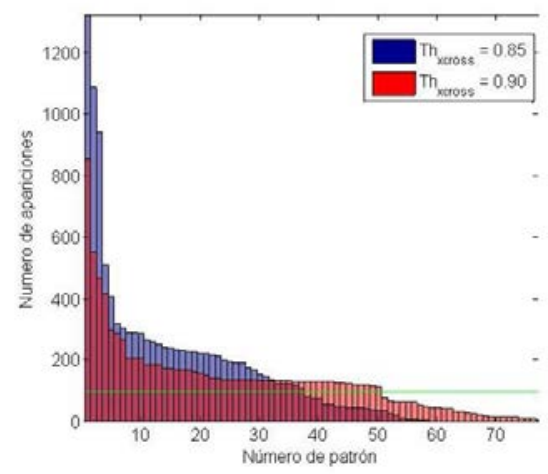

(b)

Figura 4: Patrones obtenidos con diferentes umbrales: (a) morfología del patrón más significativo con 2 umbrales distintos; (b) cantidad de patrones encontrados y frecuencia de aparición (donde la línea verde indica las 100 apariciones por encima de lo cual se considera un latido relevante)

\subsection{MODELADO DE FOURIER}

Los parámetros del modelo de Fourier para la señal ICG están descritos en la Tabla 1 y su simulación está representada en la Figura 5.

Tabla 1: Parámetros del modelo de Fourier.

\begin{tabular}{|c|c|c|c|}
\hline Parámetro & Valor & Parámetro & Valor \\
\hline$a_{1}$ & $-0,475$ & $b_{1}$ & 1,023 \\
\hline$a_{2}$ & $-0,738$ & $b_{2}$ & $-0,108$ \\
\hline$a_{3}$ & $-0,213$ & $b_{3}$ & $-0,221$ \\
\hline$a_{4}$ & 0,000 & $b_{4}$ & 0,081 \\
\hline$a_{5}$ & $-0,140$ & $b_{5}$ & 0,047 \\
\hline$a_{6}$ & $-0,138$ & $b_{6}$ & $-0,055$ \\
\hline$a_{7}$ & $-0,005$ & $b_{7}$ & $-0,075$ \\
\hline$a_{8}$ & 0,052 & $b_{8}$ & 0,013 \\
\hline$a_{9}$ & $-0,015$ & $b_{9}$ & 0,068 \\
\hline
\end{tabular}

Se ha utilizado este modelo para generar una serie temporal de mayor duración. En este primer caso, no se han introducido variaciones tiempofrecuencia, por tanto los 9 picos de frecuencia deberán localizarse en $n \omega_{0}$, tal y como se observa en la Figura 6, donde también se han aislado en el dominio de la frecuencia las diferentes componentes de la señal de ICG.

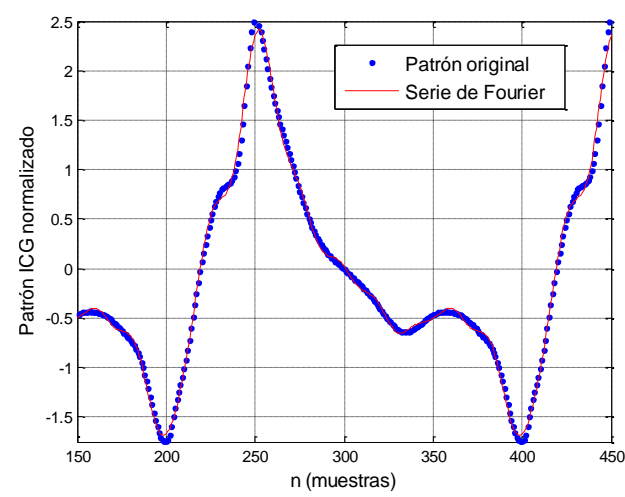

Figura 5: Modelo de Fourier de un patrón ICG

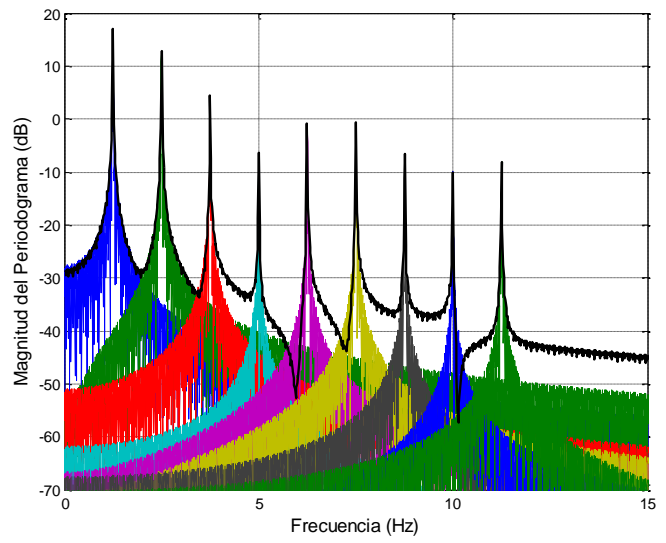

Figura 6: Periodograma de una señal ICG de frecuencia constante (negro) con su descomposición en armónicos de frecuencia (distintos colores)

\subsection{MODELADO CON VARIACIONES LINEALES Y CUADRÁTICAS EN TIEMPO- FRECUENCIA}

El modelo presentado en la Tabla 1 se ha modificado incluyendo la frecuencia $\omega(t)=2 \pi f_{i}(t)$. En el caso de una variación lineal, la señal cambia de 40 latidos por minuto (lpm) a $80 \mathrm{lpm}$ en $T=80$ segundos (20000 muestras). Por lo tanto, $\beta_{\text {lineal }}=\left(f_{1}-\right.$ $\left.f_{0}\right) / 2 t_{1}$ donde $f_{1}=40 \mathrm{lpm}, f_{2}=80 \mathrm{lpm}, t_{1}=f_{s}$. $T$ muestras. En el caso de una variación cuadrática, $\beta_{\text {cuadrática }}=\left(f_{1}-f_{0}\right) / 3 t_{1}^{2}$. La Figura 7 presenta dos señales sintéticas ICG con variaciones tiempofrecuencia lineal (a) y cuadrática (b).

Estas dos señales ICG generadas, con base tiempofrecuencia lineal y cuadrática, muestran una respuesta en frecuencia con algunas diferencias importantes a la 
mostrada en la Figura 6. Ahora, los picos principales se expanden de forma lineal (Figura 8a) o cuadrática (Figura 8b) en tiempo y en frecuencia. Esta variabilidad se traducirá en picos más amplios que interferirán entre sí en el periodograma.

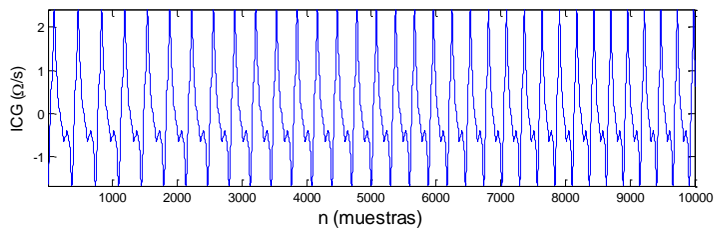

(a)

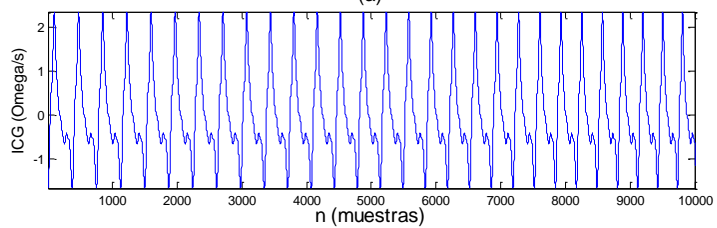

(b)

Figura 7: Señales ICG con variaciones de 40 a 80 lpm de forma (a) lineal y (b) cuadrática

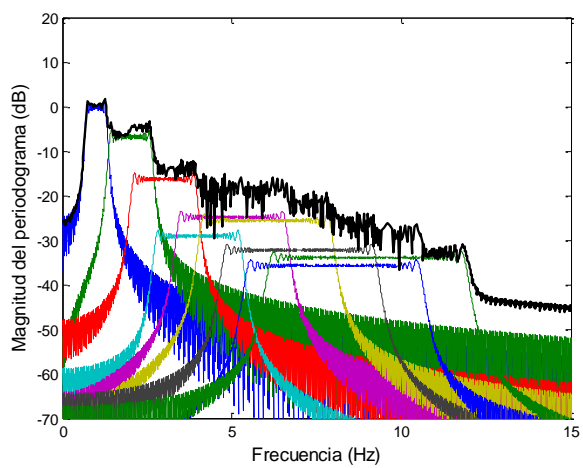

(a)

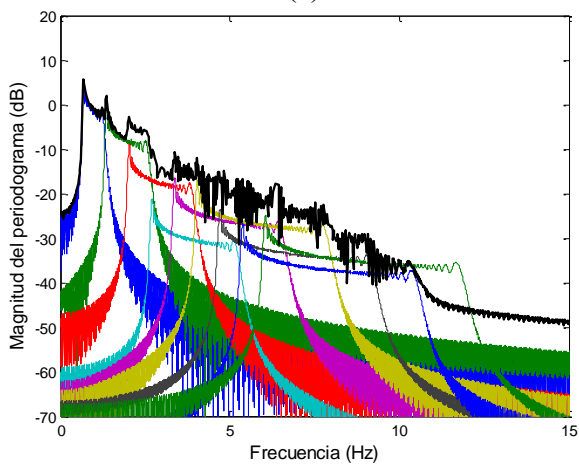

(b)

Figura 8: Periodograma (en negro) de las señales de la Figura 7 con variación lineal (a) y cuadrática

(b) con sus componentes frecuenciales individualizadas (en color)

\subsection{MODELADO CON VARIACIONES ALEATORIAS Y CAÓTICAS EN TIEMPO- FRECUENCIA}

En este caso, en lugar de modificar directamente la frecuencia de una serie de Fourier, se ha modificado la duración de cada latido según una serie cardiaca RR. En la Figura 9 se muestran series de duraciones RR distribuidas de forma uniforme (a) y de forma caótica (b) generada a partir del mapa de Hénon, con los valores iniciales $H_{x 0}=H_{y 0}=0,5$ y coeficientes $a=$ 1,4 y $b=0,3$.
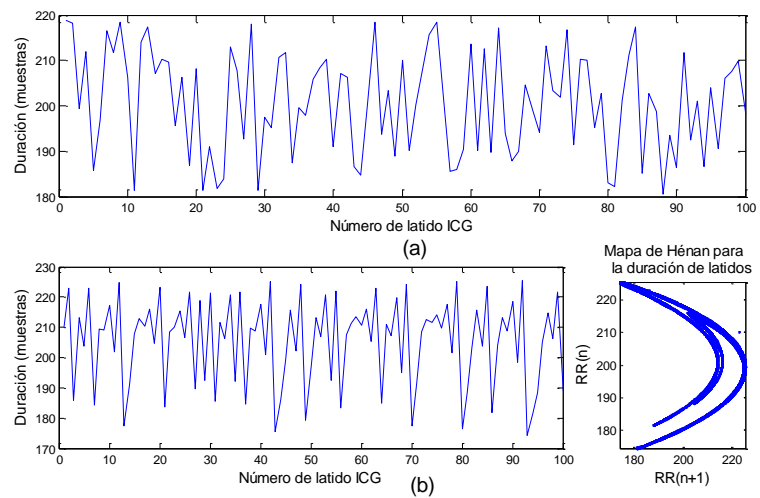

Figura 9: Series de latidos cardíacos aleatorios (a) y caóticos (b, izda.) según el mapa de Hénon dado (b, dcha.)

\subsection{MODELADO CON VARIACIONES RESPIRATORIAS}

En esta última variante de la señal ICG simulada, se han introducido simultáneamente las siguientes características:

- Variación lineal de la señal ICG de 501pm a $701 \mathrm{pm}$ y de vuelta a $501 \mathrm{pm}$.

- Influencia de la respiración con una frecuencia de 1 ciclo cada 12 latidos ICG.

- Ruido gaussiano blanco aditivo con $\mathrm{SNR}=20 \mathrm{~dB}$.

- Interferencia de red de $50 \mathrm{~Hz}$ del 5\% del valor máximo de la señal ICG.

Esta combinación tiene como objetivo sintetizar una señal ICG muy similar a una señal ICG real. Los resultados se pueden observar en la Figura 10.

\section{DISCUSIÓN}

Como se mencionó anteriormente, hay varios tipos de distribuciones tiempo-frecuencia (DTF). La decisión de qué DTF emplear depende en gran medida de las características de las señales objeto de estudio. Cuando se trabaja con señales biomédicas, la selección de la DTF más adecuada se realiza muy a menudo por comparación visual de los gráficos de las respuestas de las diferentes DTF [1]. Claramente, esto conduce a decisiones subjetivas no exentas de discrepancias en las opiniones de distintos expertos. 


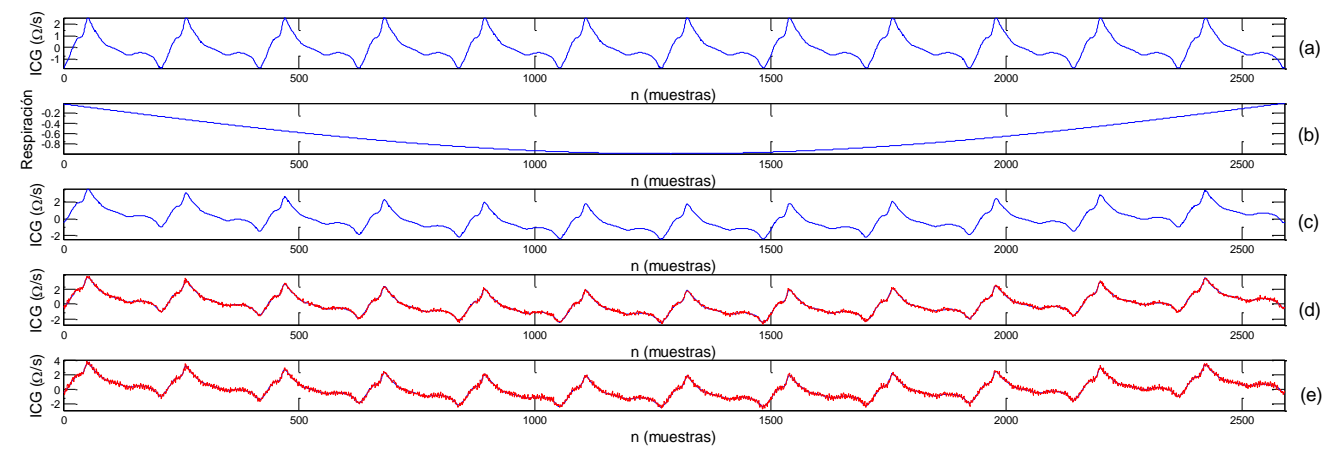

Figura 10: (a) Señal ICG sintética con frecuencia linealmente variable; (b) modulación por respiración; (c) señal (a) con la modulación (b); (d) señal (c) con ruido blanco gaussiano; (e) señal (d) con interferencia de red

Además, frecuentemente se suelen comparar distintas DTFs para las mismas señales reales. Esto implica que, con el fin de elegir la DTF ideal, el investigador debe saber y conocer perfectamente qué contenido tiempo-frecuencia espera de la señal en análisis, lo cual no siempre es factible.

Otros autores han llevado a cabo la comparación de DTFs con medidas cuantitativas que ofrecen una mayor facilidad de selección de la mejor DTF [5]. En otros estudios, se han comparado las DTFs con señales sintéticas no biomédicas, formadas por señales moduladas en frecuencia y tonos individuales [11].

La adquisición de nuevas señales biomédicas no analizadas hasta el momento (o que fueron descartadas pero se están actualmente investigando de nuevo) plantea un problema importante en la elección de la DTF idónea. Esto justifica la necesidad de crear directrices sobre cómo crear señales sintéticas biomédicas para ser estudiadas en aplicaciones DTF. En este sentido este trabajo muestra una metodología que puede aplicarse a cualquier tipo de señal antes de ser analizada con métodos de representación tiempofrecuencia.

La combinación de un análisis de reconocimiento de patrones de varias señales y el modelado de Fourier ha generado un buen resultado con baja exigencia computacional. En primer lugar, el análisis de reconocimiento de patrones se basa en un análisis de correlación y la actualización constante de la base de datos de patrones. Durante esta actualización pueden producirse ligeras variaciones en la base de datos final si cambia la ordenación de los latidos. Por otra parte, el promediado reduce el ruido de las señales patrón almacenadas en la base de datos. Sin embargo, existen dos puntos críticos. En primer lugar, los puntos de inicio y final de cada latido deben estar debidamente detectados para que los latidos se puedan comparar adecuadamente. En segundo lugar, el umbral de correlación es una medida importante en términos de rendimiento computacional. Para ello, se ha asignado el umbral más bajo posible que no introduzca diferencias relevantes respecto a los resultados obtenidos con umbrales mayores. Esto es importante para reducir el tamaño de la base de datos de patrones, reducir el número de iteraciones del algoritmo y acelerar el proceso.

El modelado del patrón definitivo se ha realizado con 9 componentes frecuenciales, tal como se ha presentado en la Figura 7 y Tabla 1. Sin embargo, siempre es recomendable utilizar la mayor cantidad de componentes frecuenciales con el fin de reducir las diferencias entre la señal original y la señal modelada. Es también importante enfatizar que un mayor número de componentes frecuenciales en la señal modelada implicará una mayor dificultad en la búsqueda del algoritmo DTF más adecuado para diferenciar entre todas las componentes frecuenciales de la señal y sus eventuales interferencias.

Debe subrayarse la variedad de las señales creadas. Se han presentado dos métodos diferentes para modificar la frecuencia fundamental del patrón ICG inicial: modificar la frecuencia de forma lineal o cuadrática y modificar la duración temporal de patrones consecutivos. Ambas metodologías proporcionan resultados satisfactorios. Además, se han implementado otras modificaciones como la adición de interferencias respiratorias o de ruido de red.

\section{CONCLUSIÓN}

Este documento detalla un procedimiento para la construcción de bases de datos de señales biomédicas sintéticas. Concretamente, se han analizado señales de impedancia cardiográfica reales con métodos sencillos y eficaces que ofrecen la posibilidad de modificar y generar señales sintéticas. Esta metodología permitirá evaluar eficazmente distintas distribuciones tiempofrecuencia, cuya bondad depende en gran medida de la señal objeto de análisis. 


\section{Agradecimientos}

Este trabajo ha sido financiado dentro del programa de doctorado industrial DI-2014 de la Generalitat de Catalunya (España).

\section{Referencias}

[1] Aimie-salleh N., Malarvili M.B., and Phillip A.C., (2015) "Quantitative Comparison of Time Frequency Distribution for Heart Rate Variability Using Performance Measure", J of Wireless Net and Com., vol. 5, pp. 1-5.

[2] Boashash, B., Azemi, G., O’Toole, J. M., (2013) "Time-frequency processing of nonstationary signals: Advanced tfd design to aid diagnosis with highlights from medical applications," IEEE Signal Process. Mag., vol. 30 , no. 6 , pp. $108-119$.

[3] Boashash, B., (2003) Time frequency signal analysis and processing: a comprehensive reference. Elsevier.

[4] Bruce, E. N., (2001) Biomedical Signal Processing and Signal Modeling. John Wiley $\&$ Sons.

[5] Chan, H.-L., Huang, H.-H., Lin, J.-L., (2001) "Time-Frequency Analysis of Heart Rate Variability During Transient Segments," Ann Biomed Eng, vol. 29, no. 11, pp. 983-996.

[6] Clariá, F., Vallverdú, M., Riba, J., Romero, S., Barbanoj, M. J., Caminal, P. (2011) "Characterization of the cerebral activity by time-frequency representation of evoked EEG potentials.," Physiol. Meas., vol. 32, no. 8, pp. 1327-1346.

[7] Clariá, F., Vallverdú, M., Baranowski, R., Chojnowska, L., Caminal, P., (2008) "Heart rate variability analysis based on timefrequency representation and entropies in hypertrophic cardiomyopathy patients.," Physiol. Meas., vol. 29, no. 3, pp. 401-416.

[8] Hénon, M., (1976) “A two-dimensional mapping with a strange attractor," Commun. Math. Phys., vol. 50, no. 1, pp. 69-77.

[9] Jospin, M., Aguilar, J. P., Gambus, P. L., Jensen, E. W., Vallverdu, M., Caminal, P., (2012) "Validation of the qCO cardiac output monitor during Valsalva maneuver," Proc. Annu. Int. Conf. IEEE Eng. Med. Biol. Soc. EMBS, pp. 240-243.
[10] Pan, Tompkins, (1985) "A real-time QRS detection algorithm," IEEE Trans. Biomed. Eng., vol. 3, pp. 230-6.

[11] Sucic, V., Boashash, B., (2001) "Parameter Selection for Optimising Time-Frequency Distributions and Measurements of TimeFrequency Characteristics of Non-Stationary Signals," Proc. IEEE Internat. Conf. Acoust. Speech Signal Process., vol. 6, pp. 35573560 . 